\title{
Genetic profiling of the 5-top cancers among Arabian populations in relation to their genealogical landscape: towards establishment of gene therapy platform in the region
}

\begin{abstract}
An account has been given to determine the prevalence of the most common 5-top cancer among males and females in Arab populations. The data available on the site of the World Health Organization (WHO) was collected officially from the respective Governments of Arabian countries which are vital and very reliable. The results show that the 5-most common cancers for the males are prostate, colorectal, lung, bladder, and non-Hodgkin lymphoma and for the women population it is breast, ovary, cervical, thyroid and non-Hodgkin lymphoma. The lowest cancer frequency among males was: bladder $(4 \%)$, lung $(3 \%)$, prostate $(1 \%)$, colorectal $(4 \%)$ and leukemia $(0.5 \%)$ while amongst the females are breast $(20 \%)$, ovary $(2 \%)$, cervical $(2 \%)$, thyroid $(4 \%)$ and lung $(1 \%)$ in the respected country. Whereas the highest frequency of cancer among males was: bladder $(19 \%)$ lung $(23 \%)$, prostrate $(10 \%)$, colorectal $(11 \%)$ and non-Hodgkin lymphoma (1\%) and for females it is breast $(37 \%)$, ovary $(5 \%)$, cervical $(28 \%)$, thyroid $(19 \%)$ and lung $(3 \%)$ as in the respected country. For genealogical analysis, the microsatellites analysis of the short tandem repeats (STRs) of Y-chromosome was conducted for a total of 1000 samples of the Arabian populations using the STRs DNA analysis. The results have been registered and several hundreds have been deposited in the International DNA Gene Bank of Y-Chromosome Haplotype Reference Database (YHRD), University of Medicine in Berlin, Germany under the Accession Numbers YA003522 and YA003523. Our results on the most common halo groups and halo types, shows that a low gene diversity and a high genetic homogeneity reflects the reality that, Arabian genetic pool is a conserved one and thus the gene-flow to the region is limited to $1-5 \%$. These results have been correlated with the outcome of the constructed online-analysis of the WHO data for Arabian populations and there was no significant difference between the prevalence of the most common cancer among Arabian populations as they have the same routs of genealogical lineages. The importance of these data on the prevalence of genealogical-cancers and the future gene therapy is discussed in this article.
\end{abstract}

Volume 3 Issue 2 - 2018

Ihsan Ali Mahasneh

Department of Biotechnology, University of Sharjah, UAE

Correspondence: Ihsan Ali Mahasneh, Department of Biotechnology, Faculty of Science, University of Sharjah, Sharjah, PO Box 27272, UAE. Previous address: Biotechnology \& Genetic Engineering Laboratory, Department of Biology, Faculty of Science, Al al-Bayt University, Mafraq city, Jordan, Email imahasneh@sharjah.ac.ae

Received: December 12, 2017| Published: March 05, 2018

\section{Introduction}

Currently, there had been a worldwide growing interest in gene therapy, being the most sustainable therapeutic approach for cancers as well as genetic diseases for upcoming 50 years and beyond..$^{1-5}$ Furthermore, several researchers have reported that we can conduct a programmable editing of a target base in genomic DNA without double-stranded DNA cleavage ${ }^{5}$ and even further to this we can conduct a programmable base editing of $A \cdot T$ to $G \cdot C$ in genomic DNA without DNA cleavage. ${ }^{4}$ A particular example of the current application of CRISPR/Cas9 gene-editing technique is to eradicate HIV/AIDS. ${ }^{6}$ The genealogical culture and lifestyle of Arabs (about 400 million) is the driving force for the consanguineous marriage as favored and respected in all their communities with a range of $20-60 \%,^{7-9}$ which is higher than the isolated and rural communities particularly among the Bedouins. The prevailing rate of the congenital genetic diseases and disorder are being directed by the genealogical land escape all over the Arabs Countries. In fact, the vast majority of their internal marriages occur within the first-cousin relationship which highly provocate the prevalence of the autosomal recessive disorders in particular. The aim of this study is to determine the genealogical-cancers profiling among Arabian populations in order to establish a gene therapy platform as a new therapeutic approach in the region which will promote the overall managements of the familial cancer disease in the region.

\section{Material and methods}

\section{Project design}

In order to track down any influence of genealogical differences and according to the actual genomic-landscape of Arabian populations, a total of (1000) blood samples were collected from all Arabian countries which have been classified into 3 blocks of regional clusters:

a) East Mediterranean-Fertile-Crescent (Jordan, Iraq, Syria, Lebanon, Palestine),

b) Gulf countries (Saudi Arabia, UAE, Oman, Kuwait, Bahrain, Qatar) and

c) North African countries (Morocco, Egypt, Libya, Algeria, Tunisia). 


\section{WHO online-analysis for cancer among arabian countries}

For the prevalence of cancers and in order to determine the 5-top cancers among Arabian populations, the WHO Online-Software data were used to conduct statistical analysis. The sets of the analysis were selected for the 3 clusters (as designed above) of the Arabian populations for both males and females cancers as compared worldwide.

\section{DNA analysis}

The microsatellites analysis of the short tandem repeats (STRs) of Y-chromosome was conducted for a total of 1000 samples of the Arabian populations for the genealogical Haplogroups and haplotypes of Y-chromosome using the STRs DNA analysis. All blood samples were collected from the most authentic Arabian tribes of Qahtanite and Adnanite-lineage which were selected for this study from various Arabian populations throughout the Arabian countries. DNA was isolated from blood samples as per the manufacture protocol of Wizard Genomic DNA Purification Kit (Cat No. A1125, Pro mega). As described in the manufacturer protocol, DNA was then amplified using the Amp Fluster Y-filer PCR amplification Kit using the Gene AmpPCR System 9700 Thermo Cycler (Applied Bio system). Subsequently, PCR products were analyzed by capillary electrophoresis on an ABI 310 Genetic Analyzer using 310 data collection software version 3.1 Data were then analyzed using the Gene Mapper ID Software version 4. Alleles were determined according to the recommendations of the DNA Commission of the International Society for Forensic Genetics. Finally, the haplogroups of each haplotype was deduced using the Athey's Haplogroups Predictor version 3.16. The final data were then stored in our local database.

\section{Results and discussion}

\section{The 5-top cancers among Arabian populations}

The results show that the 5-most common cancers for males were the prostate, colorectal, lung, bladder, non-Hodgkin lymphoma and for females it is breast, ovary, cervical, thyroid and non-Hodgkin lymphoma (Figure 1). The lowest cancer frequency among males was: bladder $(4 \%)$, lung $(3 \%)$, prostate $(1 \%)$, colorectal $(4 \%)$ and leukemia $(0.5 \%)$ whilst among females: breast $(20 \%)$, ovary $(2 \%)$, cervical $(2 \%)$, thyroid $(4 \%)$ and lung $(1 \%)$ in the respected country for (Figure 2). Whereas the highest frequency of cancer among males were: bladder $(19 \%)$ lung $(23 \%)$, prostate $(10 \%)$, colorectal $(11 \%)$, non-Hodgkin lymphoma (1\%) and for females: breast (37\%), ovary $(5 \%)$, cervical (28\%), thyroid (19\%) and lung (3\%) in the respected country for (Figure 2). These data are highly important in providing us with a better understanding of the prevalence of the most common genetic diseases and the cancers epidemiology among Arabian population for better management towards establishment of gene therapy platform in the region.

\section{Profiling of Arabian genealogical landscape}

The genealogical profiling of both Arabian haplogroups and haplotypes has been registered and several hundreds have been deposited in the International DNA Gene Bank of Y-Chromosome Haplotype Reference Database (YHRD), University of Medicine in Berlin, Germany under Accession Numbers YA003522and YA003523.
Our results on the most common haplogroups and haplotypes, shows that a low gene diversity and a high genetic homogeneity reflects the reality that Arabian gene pool is a conserved one and thus the geneflow to the region is limited which is $1-5 \%$. These results have been correlated to the statistical analysis conducted from the online-analysis of the WHO data (www.who.int.) for Arabian populations and that there was no significant difference between the prevalence of the most common cancer among Arabian populations as they have the same routs of genealogical lineages. In comparison with other populations worldwide, the cancers profile in the USA, the latest studies published by the American Cancer Society ${ }^{10}$ and the National Cancer Institute (NCI) (2017) show that the most common type of cancer on the list are breast cancer, lung cancer and prostate cancer. Surprisingly, despite the genealogical differences between Arabs and the USA genealogical profiling, our results are in accordance with the this list of common cancer types in the USA (Breast Cancer, Lung cancer, Prostate cancer, Bladder cancer, Colorectal cancer, Endometrial, Cancer, Kidney Cancer, Leukaemia, Liver, Melanoma, Non-Hodgkin Lymphoma, Pancreatic Cancer, Thyroid Cancer). Moreover, in comparison with the cancer profiling in Europe, ${ }^{11}$ the most common four types of cancers, which represent half of the overall burden of cancer in Europe are breast (female), followed by colorectal, prostate and lung, which also in accordance with our results. Obviously, the similarity between our results on the cancers among Arabian countries with those of European and USA underline the importance of international collaboration through the WHO to unify the efforts for compacting cancer worldwide. In conclusion, there was no difference on the cancer profile of Arabian populations between the 3 clusters (FertileCrescent, Gulf countries and North African) and therefore the Arabian genome is highly homogenous and conserved due to the genealogical culture which have been reserved over thousands of years.

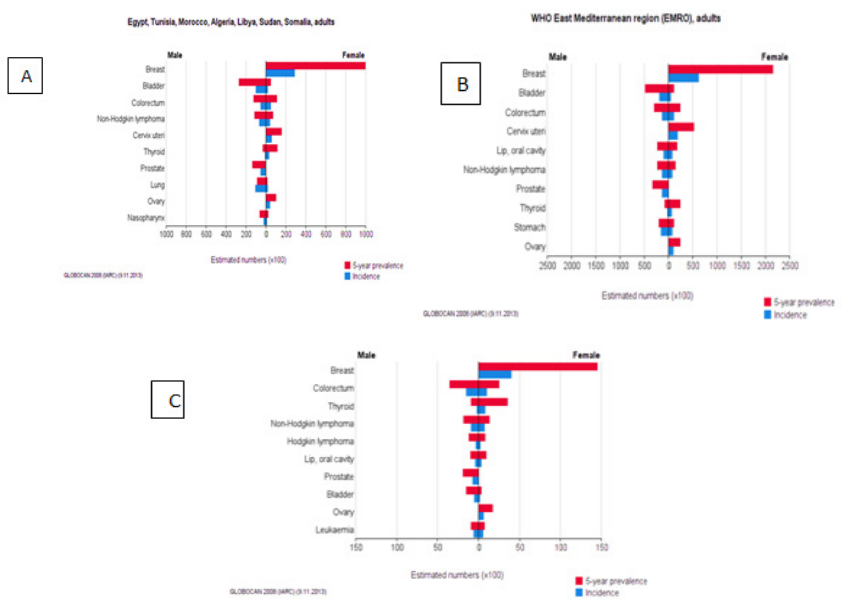

Figure I The 5-year prevalence and incidence of the most common cancer among Arabian populations: (A). Mediterranean-Fertile-Crescent (Jordan, Iraq, Syria, Lebanon, Palestine), (B). Gulf countries (Saudi Arabia, UAE, Oman, Kuwait, Bahrain, Qatar), (C). North African countries (Morocco, Egypt, Libya,Algeria, Tunisia). Statistical analysis was constructed using the online-analysis software of the WHO data (Globocan project) (www.who.int.). 


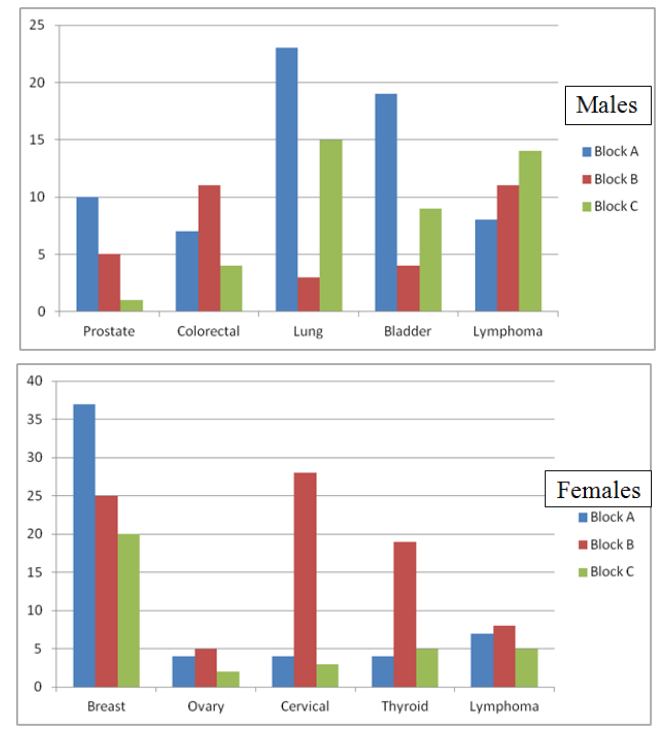

Figure 2The prevalence (\%) of the 5-Top most common cancers of males and females among Arabian populations. Block (A) of the Fertile-Crescent countries (Jordan, Iraq, Syria, Lebanon, Palestine), (b). Block (B) of the Gulf countries (Saudi Arabia, UAE, Oman, Kuwait, Bahrain, Qatar), Block (C) of the North African countries (Morocco, Egypt, Libya, Algeria, Tunisia). Statistical analysis was constructed by retrieving the public available data using the online-analysis software of the WHO data (www.who.int.).

\section{Acknowledgements}

The author would like to thank both the University of Sharjah and $\mathrm{Al}$ al-Bayt University for providing support and facilities. The author would like also to thank all those tribes and individuals for their generous donation of DNA samples which made this study possible.

\section{Conflict of interest}

The author declares no conflict of interest.

\section{References}

1. Mahasneh I. Will Molecular Biology Reshape our Near Future on 2050 \& Beyond As we are picking up the Needle from the Straw? International Journal of Molecular Biology Open Access. 2016;1(1):1-2.

2. Mahasneh I. Human Biology. University Book Shop, Sharjah, UAE. 2017.

3. Tuhin Kumar, Guha Alvan, WaiGeorg Hausner. Programmable Genome Editing Tools and their Regulation for Efficient Genome Engineering. Computational and Structural Biotechnology Journal. 2017;15:146-160.

4. Nicole M Gaudelli, Alexis C Komor, Holly A Rees, et al. Programmable base editing of $A \cdot T$ to $\mathrm{G} \cdot \mathrm{C}$ in genomic DNA without DNA cleavage. Nature. 2017;551:464-471.

5. Alexis C Komor, Yongjoo B Kim, Michael S Packer, et al. Programmable editing of a target base in genomic DNA without double-stranded DNA cleavage. Nature. 2016;533:420-424.

6. Huang Z, A Tomitaka, A Raymond et al. Current application of CRISPR Cas9 gene-editing technique to eradication of HIV/AIDS. Gene Ther. 2017;24(7):377-384.

7. Ghazi O Tadmouri, Pratibha Nair, Tasneem Obeid, et al. Consanguinity and reproductive health among Arabs. Reprod Health. 2009;6:e17.

8. Al Gazali L, Hamamy H. Consanguinity and dysmorphology in Arabs. Hum Hered. 2014;77(1-4):93-107.

9. Naamnih W, Romano Zelekha O, Kabaha A, et al. Continuous decrease of consanguineous marriages among Arabs in Israel. Am J Hum Biol. 2015;27(1):94-98.

10. Cancer Facts and Figures 2017. Atlanta: American Cancer Society; 2017.

11. Ferlay J, Steliarova Foucher E, Lortet Tieulent J, et al. Cancer incidence and mortality patterns in Europe: estimates for 40 countries in 2012. Eur J Cancer. 2013;49(6):1374-1403. 